\title{
Reciprocal effects between cognitive and affective states in women with Premenstrual Dysphoric Disorder: An Ecological Momentary Assessment study
}

\author{
Theresa Beddig $^{\mathrm{a}}$, Iris Reinhard ${ }^{\mathrm{b}}$, Ulrich Ebner-Priemer ${ }^{\mathrm{c}}$, Christine Kuehner ${ }^{\mathrm{a}, *}$ \\ ${ }^{a}$ Research Group Longitudinal and Intervention Research, Department of Psychiatry and Psychotherapy, Central Institute of Mental Health Mannheim, Medical Faculty \\ Mannheim, Heidelberg University, Germany \\ ${ }^{\mathrm{b}}$ Department of Biostatistics, Central Institute of Mental Health Mannheim, Medical Faculty Mannheim, Heidelberg University, Germany \\ ${ }^{\mathrm{c}}$ Department of Sports and Sports Science, Karlsruhe Institute of Technology, Karlsruhe, Germany
}

\section{A R T I C L E I N F O}

\section{Keywords:}

Premenstrual dysphoric disorder

Ambulatory assessment

Ecological momentary assessment

Rumination

Multilevel modeling

Lagged effects

\begin{abstract}
A B S T R A C T
Premenstrual Dysphoric Disorder (PMDD) is characterized by cyclical mood changes resulting in clinically significant distress and functional impairment. Studies on momentary cognitive and affective states and their interplay during daily life over the menstrual cycle in affected women are still lacking. Using Ecological Momentary Assessment with electronic diaries, 61 women with current PMDD and 61 healthy control women reported their current mood, rumination, and self-acceptance eight times a day over two consecutive days per cycle phase (menstrual, follicular, ovulatory, and late luteal phase). Results revealed that women with PMDD showed significant increases in negative affect and rumination and decreases in positive affect and self-acceptance toward the end of the cycle. Lagged analyses demonstrated stronger within-person reciprocal effects of cognitions and mood in PMDD women compared to controls with the effect of rumination on subsequent negative affect being limited to the late luteal phase. Identified stronger prospective associations between cognitive processes and mood deteriorations in women with PMDD suggest that affected women are more sensitive to detrimental effects of either dimension. Hence, therapeutic strategies aiming at reducing ruminative thoughts and improving self-acceptance such as mindfulness-based interventions could be promising for reducing the burden of PMDD.
\end{abstract}

\section{Introduction}

\subsection{Background}

Premenstrual Dysphoric Disorder (PMDD) is the most severe form of premenstrual burden, causing clinically significant distress and marked impairment of psychosocial functioning (Lanza di Scalea \& Pearlstein, 2019). Outlined as a new diagnostic category in DSM-5, PMDD is defined by the presence of at least five symptoms during the late luteal phase of the menstrual cycle including at least one out of four marked affective symptoms such as affective lability, irritability, depressed mood or anxiety (American Psychiatric Association, 2013). A full diagnosis requires daily symptom ratings over two symptomatic cycles, although a provisional diagnosis of PMDD can be made without (APA, 2013). While the less severe premenstrual syndrome has a prevalence of about $13-20 \%$ in community samples depending on the underlying diagnostic criteria (Beddig \& Kuehner, 2017; H. U.; Wittchen, Becker,
Lieb, \& Krause, 2002), PMDD afflicts 3-8\% of women in fertile ages (Dennerstein, Lehert, \& Heinemann, 2012; Lanza di Scalea \& Pearlstein, 2019). PMDD frequently takes a chronic course (H. U. Wittchen et al., 2002), and suicidality is increased (Owens \& Eisenlohr-Moul, 2018; Pilver, Libby, \& Hoff, 2013).

In light of the high comorbidity and symptom overlap between PMDD with Major Depression and anxiety disorders shared vulnerability factors have been proposed. One such transdiagnostic factor might be rumination, defined as the tendency to passively and repetitively analyze one's distress, problems, and concerns, without taking actions (Nolen-Hoeksema \& Watkins, 2011). Previous research showed that rumination in response to negative mood is a stable risk factor for mental disorders, especially for depression (e.g. Huffziger, Reinhard, \& Kuehner, 2009; Lyubomirsky, Layous, Chancellor, \& Nelson, 2015; Nolen-Hoeksema, Wisco, \& Lyubomirsky, 2008) but also for other common mental disorders (Nolen-Hoeksema \& Watkins, 2011). Following the transdiagnostic perspective, maladaptive cognitive

\footnotetext{
* Corresponding author. Research Group Longitudinal and Intervention Research, Central Institute of Mental Health, D-68159, Mannheim, Germany.

E-mail address: Christine.Kuehner@zi-mannheim.de (C. Kuehner).
} 
processes such as rumination could also play a role in the etiology and maintenance of PMDD. In women with PMDD, cognitive processes have been relatively understudied and if so have been dominated by investigations of respective traits or habitual coping styles. It is thought that those women suffering from premenstrual changes who have a ruminative response style may be more vulnerable to developing PMDD (cf. Craner, Sigmon, \& Martinson, 2015; Craner, Sigmon, Martinson, \& McGillicuddy, 2014), pointing toward a multifactorial model in which psychological factors interact with physiological cycle changes. In fact, former studies found that women with premenstrual disorders tend to use less helpful coping strategies such as rumination (Craner et al., 2014), behavioral impulsivity (Petersen et al., 2016), non-acceptance of emotional responses (Reuveni et al., 2016), catastrophizing (Eggert, Witthöft, Hiller, \& Kleinstäuber, 2016), or harm-avoidance (Hsu, Liu, \& Hsiao, 2007; Miller et al., 2010). There is also some evidence that trait rumination is associated with steeper increases in premenstrual depressive symptoms (Dawson et al., 2018). Furthermore, trait rumination was found to mediate the relationship between anxiety sensitivity and premenstrual distress (Sigmon, Schartel, Hermann, Cassel, \& Thorpe, 2009). A study by Craner et al. (2015) examined momentary maladaptive psychological processes showing that in response to experimentally induced negative affect (NA) women with premenstrual disorders reacted with high levels of self-focused attention. Affected women also reported higher general use of ruminative coping and selffocused attention compared to controls. The authors propose that the tendency of affected women to use a passive, emotion-focused, ruminative coping style is likely to increase emotional symptoms. Correspondingly, in a recent randomized controlled trial the use of active coping strategies was associated with symptom relief in PMDD (Weise et al., 2019).

In contrast to studies investigating trait aspects of rumination, research on moment-to-moment relationships between state cognitions and distress during daily life in women with PMDD is lacking. To study such phenomena, an Ecological Momentary Assessment (EMA) study design is most appropriate. Here, multiple real-time assessments take place during daily life, and the resulting longitudinal data series allow the investigation of variability of momentary affect and cognitions and their temporal relationship within individuals (cf. Trull \& EbnerPriemer, 2013). In the context of PMDD research, EMA also enables to study variability of such phenomena across the menstrual cycle within persons and to compare women with and without PMDD with this regard. In contrast to PMDD research, there is growing EMA-literature examining prospective effects of momentary cognitive processes on affect and vice versa in other populations. For example, momentary rumination predicted subsequent levels of NA in clinical (e.g. Kircanski, Thompson, Sorenson, Sherdell, \& Gotlib, 2018; Ruscio et al., 2015) and in community samples (e.g. Moberly \& Watkins, 2008). Naturally occurring NA was in turn found to be followed by increased levels of rumination, suggesting a reciprocal relation between these two constructs (Moberly \& Watkins, 2008). Furthermore, effects of momentary cognitive processes on positive emotions have been documented in the context of mindfulness (Garland, Geschwind, Peeters, \& Wichers, 2015; Jimenez, Niles, \& Park, 2010; Timm et al., 2018; Welz, Reinhard, Alpers, \& Kuehner, 2018). There is evidence that dispositional mindfulness is associated with higher levels of positive emotions and selfacceptance during daily life (Jimenez et al., 2010), and a recent study by Timm et al. (2018) demonstrated that mindfulness training led to improved positive affect (PA) and self-acceptance in remitted depressed patients, whereas NA and rumination decreased. Following these findings, rumination and NA as well as self-acceptance and PA appear to be tightly linked in daily life. While ruminative thinking and negative mood seem to be driven by a downward spiral especially in clinical samples (e.g. Kircanski et al., 2018; Nolen-Hoeksema et al., 2008), an upward spiral has been proposed for positive thinking and PA (Garland et al., 2015).

Given the strong affective component in PMDD, the possible role of cognitive processes in influencing affective processes is of particular interest. Since symptoms occur in a cyclical recurring pattern, it is likely that women with PMDD are especially vulnerable to dysfunctional cognitions during the late luteal phase of the menstrual cycle (cf. Read, Perz, \& Ussher, 2014). Hence, similar to studies with other clinical samples (e.g. Kircanski et al., 2018), a downward spiral of increased rumination and decreased self-acceptance exerting mood worsening and vice versa might be effective in PMDD women particularly in the late luteal phase. Using EMA in PMDD research has been repeatedly called for (see Bosman, Jung, Miloserdov, Schoevers, \& aan het Rot, 2016; Owens \& Eisenlohr-Moul, 2018), and we have implemented a first EMA study in this context (Beddig, Reinhard, \& Kuehner, 2019). Here, we could already demonstrate that women with PMDD showed heightened subjective stress reactivity towards daily life stressors particularly during the late luteal phase and a blunted activity of the hypothalamic-pituitary-adrenal axis (HPAA) across the menstrual cycle.

\subsection{Study aims}

Aims of the present paper were as follows. We first sought to examine possible menstrual cycle-related variations in affective and cognitive states in the PMDD sample by Beddig et al. (2019). It was expected that in PMDD women negative mood and rumination would be highest whereas positive mood and self-acceptance would be lowest during the late luteal phase compared to other cycle phases, while no such cycle-related effects were expected for controls. The second aim was to investigate possible reciprocal time-lagged relationships between rumination and NA as well as between self-acceptance and PA by also checking for possible cycle-dependent effects. Specifically, it was hypothesized that prospective effects of cognitive on affective states and vice versa would be stronger in PMDD women compared to controls, particularly in the late luteal phase.

\section{Method}

\subsection{Participants}

Women were recruited via local family doctors and gynecologists, flyers, social networks, and the homepage of the Central Institute of Mental Health (CIMH, for detailed information see also Beddig et al., 2019). To take part in the study, women had to fulfill the following inclusion criteria: a) age between 20 and 42, b) consistent length of menstrual cycle between 22 and 34 days, c) fulfillment of diagnostic criteria of a PMDD diagnosis based on DSM- 5 criteria (PMDD group) or exempt from any PMDD affective core symptoms (control group). Exclusion criteria were being pregnant or lactating during the last six months, a history of gynecological diseases, use of hormonal contraceptives and pharmaceutical medication, late evening or night shifts, body mass index $<18$ or $>35$, a lifetime history of psychotic or bipolar disorder, and current alcohol or substance abuse or dependence. Controls were matched regarding age and education. Initially 140 women were enrolled, of whom 18 (12.9\%, nine per group) withdrew from the study during the EMA phase. Reasons for discontinuating included inconsistencies with menstrual cycle reports $(n=14)$, severe technical problems $(n=2)$, decision to start hormonal contraceptives $(n=1)$, and positive pregnancy test $(n=1)$. Hence, the final sample consisted of 61 women diagnosed with PMDD and 61 controls. The study protocol was approved by the ethics committee of the Medical Faculty Mannheim, Heidelberg University. All participants gave written informed consent and were paid $100 €$ for completing the study.

\subsection{Ecological Momentary Assessment (EMA) procedure}

EMA was carried out using Motorola Moto G 2nd Generation smartphones with the software movisensXS, version 0.6.3658 (movisens $\mathrm{GmbH}$, Karlsruhe, Germany). EMA took place during four cycle 
phases of a menstrual cycle with two consecutive EMA-days per cycle phase. Individual calendars were prepared for each woman based on the date of her last menstruation onset and the average length of her menstruation and of her menstrual cycle. Assessments during the menstrual phase took place on the second and third day of menstruation $(M=2.95$ days, $S D=2.21)$. The follicular phase was examined on the second and third day after the end of menstruation $(M=8.61$ days, $S D=1.94)$. The ovulatory phase $(M=17.15$ days, $S D=2.0)$ was determined by a chromatographic ovulation test (gabControl hlH Ovulationsteststreifen, gabmed, Cologne) indicating a rise in luteinizing hormone levels in urine. Participants were asked to start testing a few days before the predicted ovulation until the result was positive, and then to complete the diary on the next two days. If ovulation did not occur, participants were asked to repeat the test in the following menstrual cycle. Assessments of the late luteal phase took place on the fourth and third day before the next menstruation was expected $(M=26.38$ days, $S D=3.02)$. Phases were validated according to the ovulation test and the exact time of the onset of the next menses. To avoid sequence effects, participants started EMA in different cycle phases.

There were eight assessments per day, with the first assessment taking place at 9.00 a.m. and the remaining seven assessments taking place between 10:00 a.m. and 09:30 p.m. at random time points at averaged $103 \mathrm{~min}$ apart $(S D=25.0)$ with a minimum interval of $45 \mathrm{~min}$. Each assessment was announced by an acoustic signal and took 3-4 min to complete. Participants had 5 min to respond, and assessments could be delayed by $15 \mathrm{~min}$. If participants were unable to respond or rejected the alarm, the assessment was saved as missing. After having completed the EMA days participants returned the device and were compensated for their participation.

\subsection{Structured assessment of psychopathology}

The diagnosis of PMDD was verified using the Structured Interview for DSM-IV TR PMDD (SCID-PMDD; Accortt, Bismark, Schneider, \& Allen, 2011) during the diagnostic baseline session. The SCID-PMDD includes all symptom criteria together with the required impairment criterion and the exclusion criterion of a mere exacerbation of symptoms of another disorder. The interview format is modeled after SCID-I (see below) and has shown high interrater reliability $(\kappa=0.96)$ (Accortt et al., 2011). For the PMDD group, the criteria for PMDD according to the SCID-PMDD had to be met with the diagnostic algorithm adapted for DSM-5. To avoid further participant burden, prospective daily ratings during at least two symptomatic cycles before study inclusion were not required. Control women had to be free of any PMDD affective core symptoms. Premenstrual physical symptoms were not an exclusion criterion for controls, given the fact that the majority of naturally cycling women are experiencing physical symptoms of varying degree during the late luteal and menstrual phase (Tschudin, Bertea, \& Zemp, 2010).

Other mental health comorbidities and exclusion criteria were assessed with the SCID-I Interview (H. Wittchen, Wunderlich, Gruschwitz, \& Zaudig, 1997), a psychometrically sound semi-structured interview for mental disorders. Furthermore, demographics were assessed during the baseline session together with the severity of depressive symptoms, measured with the 21-item Beck Depression Inventory (BDI-II; Beck, Steer, \& Brown, 1996) and trait anxiety measured with the 20-item Spielberger Trait Anxiety Inventory (STAI-T; Spielberger, Gorsuch, \& Lushene, 1970). Interviews were conducted by a trained research psychologist.

\subsection{Ecological Momentary Assessment (EMA) variables}

$N A$ and $P A$. At each assessment, participants responded on a 7-point Likert scale to negative and positive mood adjectives. For NA, participants were asked to rate how upset, irritated, nervous, listless, down and bored they felt and for PA how cheerful, energetic, enthusiastic, satisfied, relaxed and calm they felt. These items were derived from the Positive and Negative Affect Schedule (PANAS; Watson, Clark, \& Tellegen, 1988) and have been used in previous EMA-studies (Kuehner, Welz, Reinhard, \& Alpers, 2017; Timm et al., 2018; Welz et al., 2018).

Rumination. Rumination was assessed on a 7-point Likert scale with the item "At the moment I am stuck on negative thoughts and cannot disengage from them", capturing the uncontrollability facet of rumination (cf. Kuehner et al., 2017; Raes, Hermans, Williams, Bijttebier, \& Eelen, 2008; Timm et al., 2018).

Self-acceptance. Self-acceptance was assessed with the item "At the moment I accept myself how I am" on a Likert scale 1-7 (cf. Timm et al., 2018).

Daily stress events. To assess daily stress events subjects reported on a 7 -point bipolar scale $(-3=$ very unpleasant, $0=$ neutral, and 3 = very pleasant) the most important event between the current and the previous beep (cf. Wichers et al., 2009).

\subsection{Data analysis}

Statistical analyses were performed using multilevel models, taking into account that the present data were organized within nested levels. Analyses showed that for all dependent variables the three-level model including the day-level had a better fit than the two-level model according to fit indices (AIC and BIC) (Hox, Moerbeek, \& Van de Schoot, 2017). Therefore, for each outcome a multilevel model assuming three levels was applied with single assessments (level 1) nested within days (level 2), nested within persons (level 3). All models included random intercepts at level two and three, allowing individual baseline levels of the dependent variables to differ between persons and days. All statistical models included group status (PMDD/controls), cycle phase (menstrual, follicular, ovulatory, and late luteal) as a categorical variable, and the interaction group*menstrual cycle phase. Further we controlled for day (sampling days 1-8) to account for assessment reactivity and for time of the day to account for time-dependent variation within days in respective outcomes. For each outcome we checked whether time ${ }^{2}$ was significant, and if so retained it in the models. The quadratic time effect was only significant for NA which was highest in the mid-afternoon.

To examine prospective effects of daily life variables, lagged values were constructed for all observations, except for those representing the first response of a day. In the respective models, all main effects as well as the 2- and 3-way interactions between predictors of interest were included. The models tested the effect of the lagged predictor variable (e.g. rumination) at time $t$ on the respective outcome variable (e.g. NA) at time $t+1$, while controlling for the lagged outcome at time $t$ to account for any carryover, and for the lagged predictor aggregated at the person level at time $t$ to adjust for time invariant between-subject effects (B-S). To facilitate interpretation, predictor variables were person-mean centered prior to the analyses. This produced withinsubject (W-S) predictors that vary within, but not between individuals. Importantly, these predictors, originally measured on a continuous scale, were entered as dichotomized variables via person mean split due to the application of complex multilevel models. Dichotomization resulted in binary variables. Here, the focus of analysis was to compare worse states than usual with usual or better states (e.g. NA (W-S) $>0$ describes NA above the intraindividual average level compared to NA $(\mathrm{W}-\mathrm{S}) \leq 0$, which describes NA equal or below average level). In doing so we were able to estimate and test conditional interactions (in case of significant 3-way interactions) and to evaluate simple effects (in case of significant 2-way interactions), which is essential especially if checking for cycle-dependent changes (i.e., 3-way interactions group*cycle phase*predictor, in which interactions of each pair of variables are allowed to vary with the level of the third variable).

As an example of such a multilevel model (here: lagged rumination predicting negative affect (NA)), the level 1 model can be described as: 


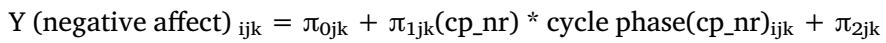
* lagged negative affect $\mathrm{ijk}_{\mathrm{j}}$

$+\pi_{3 \mathrm{jk}} *$ time of day $_{\mathrm{ijk}}+\pi_{4 \mathrm{jk}} *$ (time of day $)_{\mathrm{ijk}}^{2}+\pi_{5 \mathrm{jk}} *$ lagged rumination $(\text { dich })_{\mathrm{ijk}}$

$+\pi_{6 \mathrm{jk}}\left(\mathrm{cp}_{-} \mathrm{nr}\right) *$ cycle phase(cp_nr) $)_{\mathrm{ijk}} \mathrm{X}$ lagged rumination (di$\mathrm{ch})_{\mathrm{ijk}}+\varepsilon_{\mathrm{ijk}}$

Here, $Y_{i j k}$ represents the level of negative affect at time $i$ at assessment day $\mathrm{j}$ for person $\mathrm{k}$. The $\pi$ coefficients represent the intercept and the fixed main and interaction effects at level 1 , the $\varepsilon_{i j k}$ denote the residuals at level 1 . The level 2 model can be described as:

$\pi_{0 \mathrm{jk}}=\beta_{00 \mathrm{k}}+\beta_{01 \mathrm{k}} *$ assessment day $_{\mathrm{jk}}+\mathrm{u}_{0 \mathrm{jk}}$ (and $\pi_{1 \mathrm{jk}}=\beta_{10 \mathrm{k}}$; $\left.\pi_{2 j \mathrm{k}}=\beta_{20 \mathrm{k}} ; \pi_{3 \mathrm{jk}}=\beta_{30 \mathrm{k}} ; \pi_{2 \mathrm{jk}}=\beta_{20 \mathrm{k}} ; \ldots ; \pi_{6 \mathrm{jk}}=\beta_{60 \mathrm{k}}\right)$ with the $\mathrm{u}_{0 \mathrm{jk}}$ representing random intercepts for the assessment day $\mathrm{j}$ within person k. The level 3 model can be described as:

$\beta_{00 \mathrm{k}}=\gamma_{000}+\gamma_{001} * \operatorname{group}_{\mathrm{k}}+\gamma_{002} *$ lagged rumination $(\mathrm{B}-\mathrm{S})_{\mathrm{k}}+\mathrm{v}_{00 \mathrm{k}}$;

$\beta_{01 \mathrm{k}}=\gamma_{010} ; \beta_{10 \mathrm{k}}=\gamma_{100}+\gamma_{110} * \operatorname{group}_{\mathrm{k}} ; \beta_{20 \mathrm{k}}=\gamma_{200} ; \beta_{30 \mathrm{k}}=\gamma_{300} ;$ $\beta_{40 \mathrm{k}}=\gamma_{400}$;

$\beta_{50 \mathrm{k}}=\gamma_{500}+\gamma_{510} *$ group $_{\mathrm{k}} ; \beta_{60 \mathrm{k}}=\gamma_{600}+\gamma_{610} *$ group $_{\mathrm{k}}$

Here the $\mathrm{v}_{00 \mathrm{k}}$ indicate the random intercept for person $\mathrm{k}$. Note that we included three dummy variables for the categorical variable cycle phase, and the appendix “(cp_nr)" indicates a specific cycle phase.

To control for possible confounding effects of depressive symptom severity, trait anxiety symptoms and daily stress events, analyses were repeated by controlling for these variables.

Our hypotheses-driven main analyses investigating 3-way interactions (cycle phase*lagged predictor*group) were not Bonferroni-adjusted. In case of a nonsignificant interaction effect with cycle phase, we looked at possible significant 2-way interactions (group*lagged predictor) in the model, the latter indicating phase-independent group differences in the association between lagged predictor and outcome. Since these analyses were exploratory, respective $\mathrm{p}$-values were Bonferroni-corrected for multiple testing, as suggested by Hox et al. (2017). Similarly, all post hoc tests were Bonferroni-adjusted. All statistical analyses were performed using the statistical software IBM SPSS Version 23. The significance level was set at $\alpha=0.05$.

\section{Results}

\subsection{Compliance}

Statistical analyses were based on 122 participants (PMDD group: $\mathrm{N}=61$; control group: $\mathrm{N}=61$ ). Altogether, 6818 of 7808 possible assessments were recorded, which corresponds to an overall response rate across participants of $87.3 \%$, reflecting a high level of compliance (cf. Courvoisier, Eid, \& Lischetzke, 2012).

\subsection{Descriptives}

Descriptive information on demographic, clinical, and EMA variables are listed in Table 1 . Groups did not significantly differ with respect to age, education, marital status, work situation, percentage with children, and mean duration of the menstrual cycle. In contrast, PMDD women displayed higher BDI-II mean scores, higher trait anxiety levels, and a larger percentage had a lifetime diagnosis of depression.

\subsection{Cycle dependent variation of affect and cognitions}

To investigate whether women with and without PMDD differed in cognitive and affective states over the menstrual cycle, four separate models were calculated. As shown in Table 2, the group*cycle phase interaction was significant in predicting NA $(p<0.001)$, PA $(p<0.001)$, rumination $(p<0.001)$, and self-acceptance $(p<0.001)$. Post hoc tests using Bonferroni correction revealed higher levels of NA and rumination as well as lower levels of PA during the late luteal phase in women with PMDD compared to all other cycle phases ( $p$ 's $\leq 0.01)$ and decreased levels of self-acceptance compared to the follicular and ovulatory phase $\left(p_{\text {menstrual }}=0.164, p_{\text {follicular }}<0.001\right.$, $p_{\text {ovulatory }}<0.001$ ). In contrast, no cycle phase effect on momentary states was observed in control women (all $p$ 's $>0.55$ ). For graphical demonstration, see Fig. 1.

\subsection{Time-lagged models of predictors and outcomes}

\subsubsection{Time-lagged models of NA and rumination}

The analysis investigating lagged rumination predicting NA revealed significant time-lagged associations varying across menstrual cycle phases and groups (group* cycle phase* rumination lag $(F(3$, 4935) $=2.87, p=0.035$, see Table 3 ). Post hoc tests using Bonferroni correction revealed that in PMDD women, momentary levels of rumination (at time $t$ ) above their individual average significantly predicted a premenstrual increase in NA at the subsequent time point $(t+1)$ $(p<0.01)$, whereas for controls no menstrual cycle-related effect was observed (all $p$ 's $>0.37$ ) (see Fig. 2 A).

The analysis investigating lagged NA predicting rumination revealed no significant three-way interaction group* cycle phase* NA lag $(F(3,5270)=0.24, p=0.868)$. However, we identified a significant group* NA lag effect $(F(1,5242)=7.42, p=0.018$ (Bonferroni-corrected), see Table 3). Fig. 2 B indicates that in PMDD women momentary NA levels (at time t) above average resulted in a stronger increase in momentary rumination at the subsequent time point $(t+1)$ compared to controls regardless of cycle phase. Post hoc tests revealed that for both groups the prospective effect of NA on rumination was significant (PMDD: $p<0.001$, controls: $p<0.001$ ).

\subsubsection{Time-lagged models of PA and self-acceptance}

The analysis investigating lagged self-acceptance predicting PA revealed no significant effect of group*cycle phase*self-acceptance lag $(F$ $(3,2779)=0.49, p=0.689)$. We identified a significant interaction group*self-acceptance lag $(F(1,44400)=7.86, p=0.015$ (Bonferronicorrected), see Table 4). Fig. $2 \mathrm{C}$ indicates that in PMDD women momentary levels of self-acceptance below average (at time $t$ ) resulted in a stronger decrease in PA at the subsequent time point $(t+1)$ compared to controls regardless of cycle phase. Post hoc tests revealed that the prospective effect of self-acceptance on PA was only significant in women with PMDD ( $p<0.001)$, not in controls $(p=0.936)$.

While the analysis investigating lagged PA predicting self-acceptance revealed no significant three-way interaction group*cycle phase* PA lag $(F(3,5212)=0.90, p=0.438)$, the interaction group* PA lag demonstrated a significant effect on self-acceptance $(F(1$, $5201)=16.70, p<0.001$ (Bonferroni-corrected), see Table 4). Fig. 2 $\mathrm{D}$ indicates that in PMDD women momentary levels of PA below average (at time $t$ ) resulted in a stronger decrease in self-acceptance to the subsequent time point $(t+1)$ compared to controls regardless of cycle phase. Post hoc tests revealed that the prospective effect of PA on self-acceptance was only significant in women with PMDD $(p<0.001)$, not in controls $(p=0.147)$.

\subsubsection{Confounder analysis}

Including the severity of depressive symptoms (BDI-II), anxiety symptoms (STAI-T) and daily stress events as covariates did not change any of the reported effects. Therefore, the present results were not affected by possible group differences in depressive and anxiety symptom severity and daily stress events. 
Table 1

Descriptions of women with PMDD and controls.

\begin{tabular}{|c|c|c|c|c|}
\hline & $\operatorname{PMDD}(n=61) \% / M(S D)$ & Controls $(n=61) \% / M(S D)$ & Test statistic & $p$ \\
\hline \multicolumn{5}{|l|}{ Demographic Variables } \\
\hline Age & $29.4(5.8)$ & $29.5(5.1)$ & $\mathrm{t}=-0.03$ & .977 \\
\hline Education (\% with high school degree & $72.1 \%$ & $75.4 \%$ & $\mathrm{Chi}^{2}=0.17$ & .681 \\
\hline Work Situation (\% in regular job or education) & $80.3 \%$ & $90.2 \%$ & $\mathrm{Chi}^{2}=2.35$ & .126 \\
\hline Marital Status (\% married or living together) & $60.7 \%$ & $59.0 \%$ & $\mathrm{Chi}^{2}=0.03$ & .853 \\
\hline Children (\%) & $24.6 \%$ & $26.2 \%$ & $\mathrm{Chi}^{2}=0.04$ & .835 \\
\hline BMI & $23.6(4.1)$ & 23.5(4.3) & $\mathrm{t}=0.12$ & .903 \\
\hline \multicolumn{5}{|l|}{ Clinical Variables } \\
\hline Lifetime Diagnosis of Depression (SCID-I) & $54.1 \%$ & $21.3 \%$ & $\mathrm{Chi}^{2}=13.96$ & $<.001$ \\
\hline BDI-II ${ }^{\mathrm{a}}$ score at baseline & $10.9(8.9)$ & $4.8(5.6)$ & $\mathrm{t}=4.53$ & $<.001$ \\
\hline STAI-T $^{\mathrm{b}}$ score at baseline & $45.6(11.3)$ & $37.2(8.6)$ & $\mathrm{t}=4.61$ & $<.001$ \\
\hline \multicolumn{5}{|l|}{ EMA $^{\mathrm{c}}$ Variables } \\
\hline Compliance Rate & $86.6 \%$ & $88.0 \%$ & $\mathrm{Chi}^{2}=33.62$ & .071 \\
\hline Duration (in days) of Menstrual Cycle during EMA & $29.0(3.1)$ & $29.4(3.7)$ & $\mathrm{t}=-0.77$ & .444 \\
\hline Duration (in days) of Period during EMA & $5.3(1.1)$ & $5.6(1.7)$ & $\mathrm{t}=-0.85$ & .399 \\
\hline
\end{tabular}

${ }^{\text {a }}$ BDI-II $=$ Beck Depression Inventory-Revised.

b STAI-T $=$ Spielberger Trait Anxiety Inventory.

${ }^{\mathrm{c}}$ EMA $=$ Ecological Momentary Assessment.

\section{Discussion}

\subsection{Summary of results}

The purpose of the current EMA study was to explore menstrual cycle-related variations of affective and cognitive states during daily life and to examine time-lagged associations between these states in women with PMDD and healthy controls. While affective symptoms of low mood, decreased interest, irritability, and anxiety occur as part of many mental disorders, the prominent feature that distinguishes PMDD from similar entities is its time course, with the late luteal phase confinement of symptoms (APA, 2013). In this regard, our EMA data prospectively collected over the menstrual cycle and analyzed with multilevel models - show that women with PMDD exhibited the highest NA and the lowest PA in the late luteal phase, thereby clearly confirming a symptomatic state of mood deterioration during this phase in a naturalistic, real-time context in the present PMDD sample. Moreover, PMDD women also reported highest levels of rumination and lowest levels of self-acceptance during the late luteal phase. In contrast, healthy women did not show any cycle-dependent variation of mood and cognitions.

Lagged models revealed that especially in the late luteal phase PMDD women reacted to high levels of rumination with increased levels of NA. Therefore, intensive rumination seems to have a particular mood-impairing effect toward the end of the cycle in these women. Previous studies have indicated that habitual rumination is associated with experiencing premenstrual distress (Craner et al., 2014; Dawson et al., 2018). By focusing on momentary within-person associations this study adds significantly to existing research by showing that particularly in the late luteal phase intraindividual high levels of rumination predicted deterioration in NA in women with PMDD. Our results further indicate that in PMDD women increased NA levels predicted a stronger subsequent increase in rumination compared to controls regardless of cycle phase. This adds to previous research examining habitual coping styles in the context of premenstrual disorders (e.g. Craner et al., 2015, 2014; Petersen et al., 2016; Reuveni et al., 2016). By focusing on state cognitions the present study shows that women with PMDD tend to ruminate in response to negative affective states more strongly than nonaffected women across the menstrual cycle, thereby pointing toward a trait-like characteristic of ruminative responses to negative affect in PMDD.

Taking both lagged paths together our findings suggest a reciprocal relationship between rumination and NA especially in the late luteal phase. Following the transdiagnostic perspective of rumination as a dysfunctional key mechanism in mental disorders (e.g. Lyubomirsky et al., 2015; Nolen-Hoeksema et al., 2008; Nolen-Hoeksema \& Watkins, 2011) our findings align with this concept by demonstrating a characteristic cycle-dependency in the context of PMDD.

Moreover, momentary levels of low self-acceptance resulted in a stronger decrease in PA and vice versa in PMDD women compared to controls. These observations further indicate a stronger sensitivity of affected women for the effects of negative or lack of positive self-referential thoughts. Again, we did not identify cycle-dependency for these associations, thereby pointing toward a more general underlying vulnerability of negative self-referential thoughts that could make women also more likely to developing PMDD. Importantly, controlling for daily stress events, depressive symptom severity as well as for trait anxiety levels did not affect the study results. Therefore, although comorbidity with lifetime depression was high, our study suggests that PMDD characteristics uniquely contributed to the present findings.

Table 2

Fixed effects of menstrual cycle phase and group on negative affect, positive affect, rumination, and self-acceptance.

\begin{tabular}{|c|c|c|c|c|c|c|c|c|}
\hline \multirow[t]{2}{*}{ Outcome } & \multicolumn{4}{|c|}{ Menstrual Cycle Phase } & \multicolumn{4}{|c|}{ Group * Cycle Phase } \\
\hline & Group & Menstrual & Follicular & Ovulatory & Late Luteal & $d f$ & $\mathrm{~F}$ & $p$ \\
\hline \multirow[t]{2}{*}{ Negative Affect } & PMDD & $2.8 \pm 1.2$ & $2.4 \pm 1.1$ & $2.6 \pm 1.1$ & $3.2 \pm 1.2$ & $(3,836)$ & 20.07 & $<.001$ \\
\hline & Controls & $2.1 \pm 1.1$ & $2.1 \pm 1.1$ & $2.2 \pm 1.2$ & $2.2 \pm 1.1$ & & & \\
\hline \multirow[t]{2}{*}{ Positive Affect } & PMDD & $3.8 \pm 1.3$ & $4.2 \pm 1.2$ & $4.2 \pm 1.2$ & $3.5 \pm 1.1$ & $(3,845)$ & 15.87 & $<.001$ \\
\hline & Controls & $4.4 \pm 1.2$ & $4.5 \pm 1.1$ & $4.4 \pm 1.2$ & $4.3 \pm 1.2$ & & & \\
\hline \multirow[t]{2}{*}{ Rumination } & PMDD & $2.5 \pm 1.5$ & $2.1 \pm 1.4$ & $2.2 \pm 1.51 .9 \pm 1.3$ & $2.8 \pm 1.7$ & $(3,830)$ & 15.80 & $<.001$ \\
\hline & Controls & $1.8 \pm 1.1$ & $1.9 \pm 1.3$ & & $1.9 \pm 1.3$ & & & \\
\hline \multirow[t]{2}{*}{ Self-Acceptance } & PMDD & $4.7 \pm 1.6$ & $5.2 \pm 1.5$ & $5.1 \pm 1.45 .5 \pm 1.4$ & $4.5 \pm 1.5$ & $(3,840)$ & 18.92 & $<.001$ \\
\hline & Controls & $5.6 \pm 1.3$ & $5.5 \pm 1.3$ & & $5.5 \pm 1.4$ & & & \\
\hline
\end{tabular}

Note. Models included random intercepts at level 2 and 3 as well as fixed effects for time, time ${ }^{2}$ (if significant), day, group, and cycle phase. 

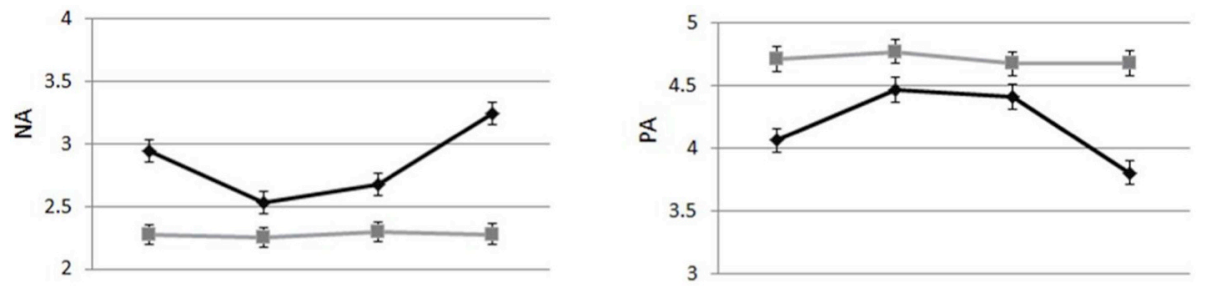

Fig. 1. Estimated mean scores of daily life variables (NA: negative affect; PA: positive affect; RUM: rumination; SA: self-acceptance) over the menstrual cycle for women with PMDD and controls. Note. Error bars represent standard error of the mean. Models include random intercepts at level 2 and 3 as well as fixed effects for time, time ${ }^{2}$ (if significant), assessment day, group, and cycle phase.
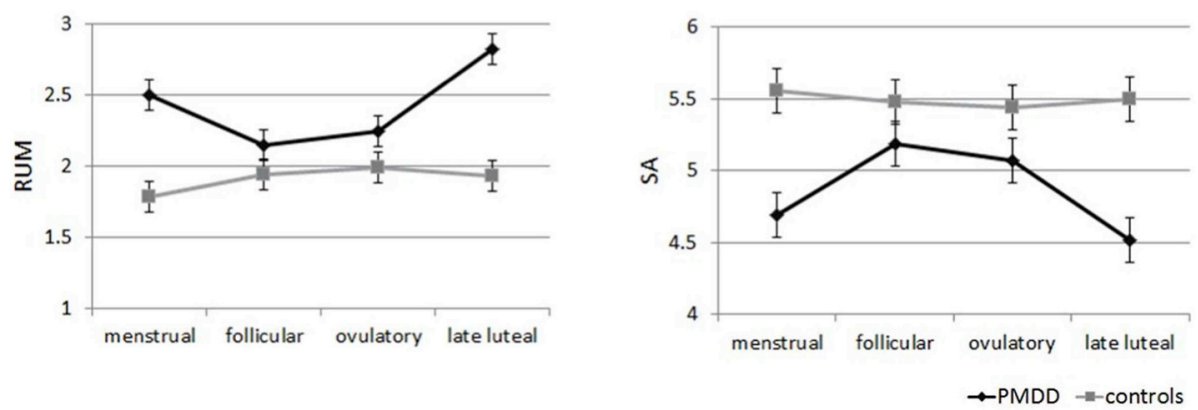

Table 3

Time-lagged models of negative affect and rumination.

\begin{tabular}{|c|c|c|c|}
\hline & \multicolumn{3}{|c|}{ 3-way Interaction Model } \\
\hline & df & $\mathrm{F}$ & $p$ \\
\hline \multicolumn{4}{|l|}{ Outcome Negative Affect $t+1$} \\
\hline \multicolumn{4}{|l|}{ Fixed effects } \\
\hline Intercept & $(1,160)$ & 90.33 & $<.001$ \\
\hline Group $^{\mathrm{a}}$ & $(1,121)$ & 13.75 & $<.001$ \\
\hline Cycle phase $^{\mathrm{b}}$ & $(3,585)$ & 16.05 & $<.001$ \\
\hline Negative Affect (W-S) (t) & $(1,4814)$ & 355.84 & $<.001$ \\
\hline Rumination (B-S) & $(1,119)$ & 167.01 & $<.001$ \\
\hline Rumination $(\mathrm{W}-\mathrm{S})(\mathrm{t})^{\mathrm{c}}$ & $(1,5257)$ & .25 & .618 \\
\hline Group * Cycle phase & $(3,588)$ & 16.86 & $<.001$ \\
\hline Rumination * Group & $(1,5306)$ & .11 & .746 \\
\hline Rumination * Cycle phase & $(3,4942)$ & .55 & .648 \\
\hline Rumination * & $(3,4935)$ & 2.87 & .035 \\
\hline \multicolumn{4}{|l|}{ Group * Cycle phase } \\
\hline \multicolumn{4}{|l|}{ Outcome Rumination $t+1$} \\
\hline \multicolumn{4}{|l|}{ Fixed effects } \\
\hline Intercept & $(1,128)$ & 3.81 & .053 \\
\hline Group $^{\mathrm{a}}$ & $(1,118)$ & .24 & .624 \\
\hline Cycle phase $^{\mathrm{b}}$ & $(3,5215)$ & 9.82 & $<.001$ \\
\hline Rumination $(\mathrm{W}-\mathrm{S})(\mathrm{t})^{\mathrm{c}}$ & $(1,5207)$ & 168.78 & $<.001$ \\
\hline Negative Affect (B-S) & $(1,117)$ & 168.19 & $<.001$ \\
\hline Negative Affect $(\mathrm{W}-\mathrm{S})(\mathrm{t})^{\mathrm{d}}$ & $(1,5244)$ & 56.77 & $<.001$ \\
\hline Group * Cycle phase & $(3,5216)$ & 12.68 & $<.001$ \\
\hline Negative Affect * Group & $(1,5242)$ & 7.42 & $.006^{\mathrm{e}}$ \\
\hline Negative Affect * Cycle phase & $(3,5271)$ & 1.43 & .233 \\
\hline Negative Affect * Group * Cycle phase & $(3,5270)$ & .24 & .868 \\
\hline
\end{tabular}

Note. W-S: within-subject (person mean-centered). B-S: between-subject. Models include random intercepts at level 2 and 3 as well as fixed effects for time, time ${ }^{\mathrm{b}}$ (if significant), and assessment day. Time $\mathrm{t}+1$ denotes the following prompt.

a Reference category: Controls.

b Reference category: Late luteal phase.

c Reference category: Rumination (W-S) t $>0$.

$\mathrm{d}$ Reference category: Negative affect $(\mathrm{W}-\mathrm{S}) \mathrm{t}>0$.

e Before Bonferroni correction.

In conclusion, our study points to the relevance of assessing affective and cognitive processes by EMA in real world settings to be able to demonstrate heightened vulnerability in women with PMDD towards worsening of their mood and towards increased negative and decreased positive self-referential thinking during the late luteal phase of their menstrual cycle. Furthermore, EMA enabled us to identify phase-specific and phase-unspecific reciprocal associations between dysfunctional momentary affective and cognitive states in respective within- person associations in these women. In general, our findings also strengthen previous research highlighting the role of psychological factors in premenstrual disorders (e.g. Craner, Sigmon, \& Young, 2016; Kleinstauber et al., 2016; Reuveni et al., 2016; Weise et al., 2019).

\subsection{Future perspectives and clinical implications}

This is likely the first EMA study assessing menstrual cycle-related variations of cognitive and affective daily life processes and their interplay in women with PMDD. In this context, EMA can clearly improve PMDD research due to higher ecological validity of assessed phenomena compared to retrospectively assessed clinical symptoms. EMA also typically covers more basic affective and cognitive features and may therefore provide greater sensitivity for connecting psychological with biological processes (cf. Conner \& Barrett, 2012; Huffziger et al., 2013). Furthermore, EMA appears to be optimally suited to capture between-as well as within-person variability in momentary states and their interplay across the menstrual cycle, which is particularly important for studying premenstrual disorders (cf. Bosman et al., 2016; Owens \& Eisenlohr-Moul, 2018).

Study findings might be relevant for therapeutic perspectives in PMDD. Results revealed that women with PMDD seem to be more prone of using rumination as a trait-like emotion regulation strategy to deal with negative affect states across cycle phases, whereas high intraindividual rumination levels seem to trigger affect deterioration particularly in the late luteal phase. Hence, rumination, identified as transdiagnostic risk factor for a series of mental disorders (cf. NolenHoeksema \& Watkins, 2011) may also be a potential therapeutic target for reducing the burden of PMDD. In this context mindfulness based interventions appear to be promising (cf. Petersen et al., 2016). For example a non-clinical study by Lustyk, Gerrish, Douglas, Bowen, and Marlatt (2011) found that high dispositional mindfulness was linked to less premenstrual symptoms, and studies with other clinical samples (e.g. Garland et al., 2015; Timm et al., 2018) showed that mindfulness training reduced negative and enhanced positive daily life cognitions such as momentary rumination and self-acceptance, and improved affect. However, methodological sound randomized controlled trials examining mindful interventions in women with PMDD are still lacking, which might be an important purpose for future research. In parallel, a first randomized controlled trial examining internet-based cognitivebehavioral therapy for PMDD by Weise et al. (2019) demonstrated that active coping when dealing with premenstrual symptoms predicted better treatment outcome, thereby underlining the importance to address coping styles. Clearly, further research is warranted to assess 

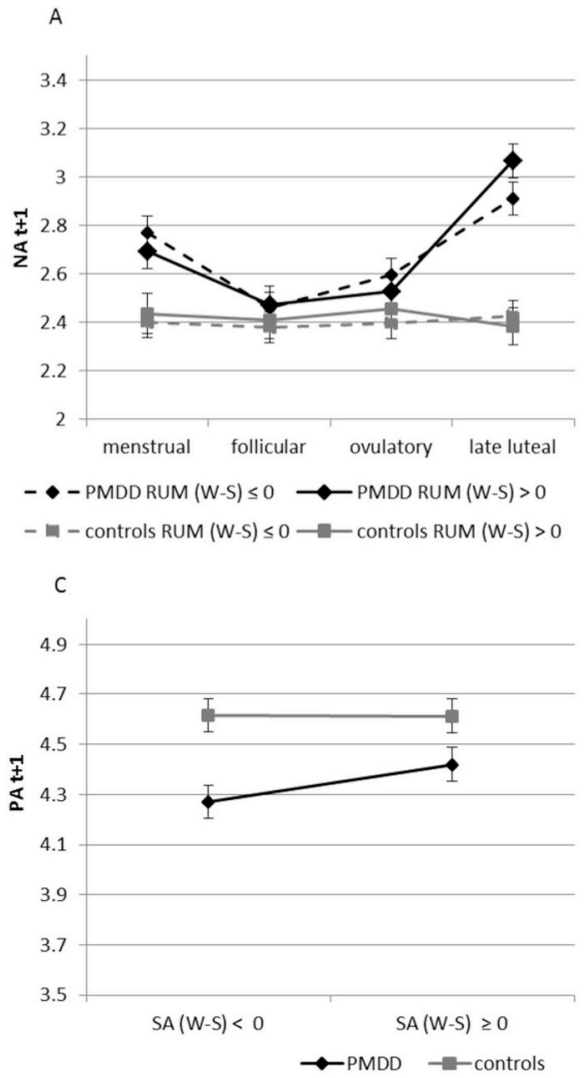

Table 4

Time-lagged models of positive affect and self-acceptance.

\begin{tabular}{|c|c|c|c|}
\hline & \multicolumn{3}{|c|}{ 3-way Interaction Model } \\
\hline & df & $\mathrm{F}$ & $p$ \\
\hline \multicolumn{4}{|l|}{ Outcome Positive Affect $t+1$} \\
\hline \multicolumn{4}{|l|}{ Fixed effects } \\
\hline Intercept & $(1,128)$ & 124.89 & $<.001$ \\
\hline Group $^{\text {a }}$ & $(1,120)$ & 8.07 & .005 \\
\hline Cycle phase $^{\mathrm{b}}$ & $(3,486)$ & 13.73 & $<.001$ \\
\hline Positive Affect (W-S) (t) & $(1,4758)$ & 372.84 & $<.001$ \\
\hline Self-acceptance (B-S) & $(1,120)$ & 125.69 & $<.001$ \\
\hline Self-acceptance $(\mathrm{W}-\mathrm{S})(\mathrm{t})^{\mathrm{c}}$ & $(1,4886)$ & 5.80 & .016 \\
\hline Group * Cycle phase & $(3,487)$ & 8.61 & $<.001$ \\
\hline Self-acceptance * Group & $(1,4440)$ & 7.86 & $.005^{\mathrm{a}}$ \\
\hline Self-acceptance $*$ Cycle phase & $(3,2797)$ & 2.39 & .067 \\
\hline Self-acceptance * Group * Cycle phase & $(3,2779)$ & .49 & .689 \\
\hline \multicolumn{4}{|l|}{ Outcome Self-acceptance $t+1$} \\
\hline \multicolumn{4}{|l|}{ Fixed effects } \\
\hline Intercept & $(1,120)$ & .20 & .656 \\
\hline Group $^{\mathrm{a}}$ & $(1,119)$ & .02 & .894 \\
\hline Cycle phase $^{\mathrm{b}}$ & $(3,5199)$ & 7.23 & $<.001$ \\
\hline Self-acceptance $(\mathrm{W}-\mathrm{S})(\mathrm{t})^{\mathrm{c}}$ & $(1,5197)$ & 789.63 & $<.001$ \\
\hline Positive Affect (B-S) & $(1,119)$ & 133.12 & $<.001$ \\
\hline Positive Affect (W-S) $(\mathrm{t})^{\mathrm{d}}$ & $(1,5200)$ & 31.79 & $<.001$ \\
\hline Group * Cycle phase & $(3,5199)$ & 11.05 & $<.001$ \\
\hline Positive Affect * Group & $(1,5201)$ & 16.70 & $<.001^{\mathrm{e}}$ \\
\hline Positive Affect * Cycle phase & $(3,5212)$ & 2.35 & .071 \\
\hline Positive Affect * Group * Cycle phase & $(3,5212)$ & .90 & .438 \\
\hline
\end{tabular}

Note. W-S: within-subject (person mean-centered). B-S: between-subject. Models include random intercepts at level 2 and 3 as well as fixed effects for time, and assessment day. Time $t+1$ denotes the following prompt.

a Reference category: Controls.

b Reference category: Late luteal phase.

${ }^{c}$ Reference category: Self acceptance $(\mathrm{W}-\mathrm{S}) \mathrm{t} \geq 0$.

${ }^{d}$ Reference category: Positive affect (W-S) $t \geq 0$.

e Before Bonferroni correction.

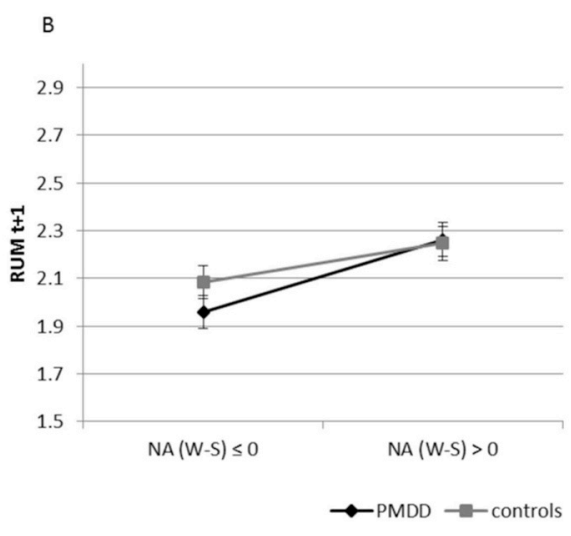

Fig. 2. Estimated mean values of the time-lagged outcomes negative affect (NA, with predictor rumination (RUM), Fig. 2 A) and RUM (with predictor NA, Fig. 2 B) as well as time-lagged outcomes positive affect (PA, with predictor self-acceptance (SA), Fig. 2 C) and SA (with predictor PA, Fig. 2 D) for women with PMDD and controls. Note. Error bars represent standard error of the mean. W-S: withinsubject (person mean-centered). Models include time, time ${ }^{2}$ (if significant), assessment day, group, cycle phase, lagged outcome and lagged predictor aggregated at the person level as covariates.

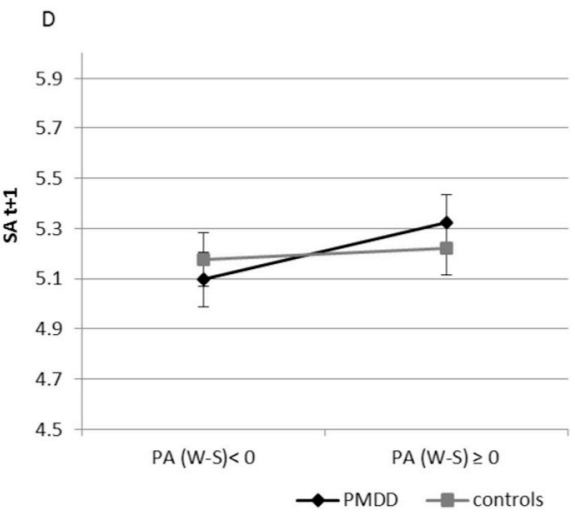

which interventions are effective in women with PMDD. Moreover, longitudinal research would aid in identifying predictive effects of momentary cognitive and affective processes and their interplay for the clinical course of PMDD, which could, in turn, provide targeting aims for therapy.

\subsection{Strengths and limitations}

Strengths of the current study include the investigation of a relatively large PMDD sample together with a control sample strictly matched regarding age and education level, the intensive longitudinal EMA-design covering all four cycle phases, the validation of the ovulation phase by a chromatographic ovulation test and the focus on temporal within-person processes to understand the momentary relationships between cognitive and affective states.

The study also has some limitations. First, given the exclusion of antidepressant and hormonal treatments, which are currently the most frequent treatments for PMDD (Epperson et al., 2012; Marjoribanks, Brown, O'Brien, \& Wyatt, 2013) the results might not be representative of all women with PMDD. Second, even though overall compliance was high and comparable between groups, nine women per group (12.9\%) dropped out due to different reasons. This could have led to a sample of more highly motivated women. Third, we did not apply the criterion of confirmation of daily symptom ratings during two consecutive symptomatic cycles prior to study inclusion. The reason for this was to reduce participant burden in this type of intensive repeated random measurement design. Thus, the diagnosis of PMDD must be considered provisional. However, prevalence rates of moderate to severe premenstrual symptoms derived from retrospective epidemiological studies have been found to be consistent with those using prospective ratings (Cunningham, Yonkers, O'Brien, \& Eriksson, 2009). Fourth, model complexity of multilevel models resulted in dichotomizing the main time-lagged variables of interest, which may have led to reduced explanatory variance. Fifth, to further increase the accuracy of 
conclusions, it would be beneficial to apply the EMA protocol over two or more consecutive menstrual cycles, which was not possible in the present study due to limited resources and may also provoke participant burden. Sixth, the EMA items were administered in a fixed order, thus a potential inflation of their associations cannot be excluded. Seventh, our study design does not allow to determine causal pathways between PMDD and daily life experiences. So it is unclear whether strong timelagged associations between cognitive and affective daily life variables represent a vulnerability factor for developing PMDD or are rather the consequence of the disorder. Here, clearly more longitudinal research is warranted. A further limitation refers to the assessment of only two days per cycle phase. Although a regular cycle was a premise to take part in the study, we sometimes might have missed days with the highest impairment. Finally, we did not assess the content of ruminative thoughts. Ruminating about symptoms might be especially crucial for affect deteriorations in women with PMDD (cf. Craner et al., 2014). Further research might address these aspects as well.

\subsection{Conclusions}

This EMA-study examined cycle-related variations of momentary cognitive and affective characteristics and their time-lagged associations in women with PMDD. Findings suggest stronger associations between cognitive (rumination and self-acceptance) and mood variables (NA and PA) in affected women compared to controls. These findings highlight the role of cognitive processes in everyday settings and may have important implications for interventions aimed at preventing and treating PMDD.

\section{CRediT authorship contribution statement}

Theresa Beddig: Investigation, Data curation, Methodology, Formal analysis, Writing - original draft, Writing - review \& editing. Iris Reinhard: Methodology, Formal analysis, Writing - review \& editing. Ulrich Ebner-Priemer: Methodology, Writing - review \& editing. Christine Kuehner: Conceptualization, Methodology, Formal analysis, Writing - review \& editing.

\section{Declaration of competing interest}

None.

\section{Acknowledgements}

This study was supported by a grant from the Deutsche Forschungsgemeinschaft to CK (DFG KU1464/6-1).

\section{References}

Accortt, E. E., Bismark, A., Schneider, T. R., \& Allen, J. J. (2011). Diagnosing premenstrual dysphoric disorder: The reliability of a structured clinical interview. Archives of Women's Mental Health, 14(3), 265-267. https://doi.org/10.1007/s00737011-0209-3.

American Psychiatric Association. (2013). (5th ed.). Diagnostic and statistical manual of mental disordersWashington, DC: DSM-5 (APA).

Beck, A. T., Steer, R. A., \& Brown, G. K. (1996). Beck depression inventory-II. San Antonio, $78(2), 490-498$.

Beddig, T., \& Kuehner, C. (2017). [Current aspects of premenstrual dysphoric disorder-a review]. Psychotherapie, Psychosomatik, Medizinische Psychologie, 67(12), 504-513. https://doi.org/10.1016/j.psyneuen.2019.104372.

Beddig, T., Reinhard, I., \& Kuehner, C. (2019). Stress, mood, and cortisol during daily life in women with Premenstrual Dysphoric Disorder (PMDD). Psychoneuroendocrinology, 109, 104372. https://doi.org/10.1016/j.psyneuen.2019.104372.

Bosman, R. C., Jung, S. E., Miloserdov, K., Schoevers, R. A., \& aan het Rot, M. (2016). Daily symptom ratings for studying premenstrual dysphoric disorder: A review. Journal of Affective Disorders, 189, 43-53. https://doi.org/10.1016/j.jad.2015.08. 063.

Conner, T. S., \& Barrett, L. F. (2012). Trends in ambulatory self-report: The role of momentary experience in psychosomatic medicine. Psychosomatic Medicine, 74, 327-337. https://doi.org/10.1097/PSY.0b013e3182546f18.
Courvoisier, D. S., Eid, M., \& Lischetzke, T. (2012). Compliance to a cell phone-based ecological momentary assessment study: The effect of time and personality characteristics. Psychological Assessment, 24(3), 713. https://doi.org/10.1037/a0026733.

Craner, J. R., Sigmon, S. T., \& Martinson, A. A. (2015). Self-focused attention in response to laboratory stressors among women with premenstrual disorders. Archives of Women's Mental Health, 18(4), 595-606. https://doi.org/10.1007/s00737-0150505-4.

Craner, J. R., Sigmon, S. T., Martinson, A. A., \& McGillicuddy, M. L. (2014). Premenstrual disorders and rumination. Journal of Clinical Psychology, 70(1), 32-47. https://doi. org/10.1002/jclp.22007.

Craner, J. R., Sigmon, S. T., \& Young, M. A. (2016). Self-focused attention and symptoms across menstrual cycle phases in women with and without premenstrual disorders. Cognitive Therapy and Research, 40(1), 118-127. https://doi.org/10.1007/s10608015-9721-5.

Cunningham, J., Yonkers, K. A., O'Brien, S., \& Eriksson, E. (2009). Update on research and treatment of premenstrual dysphoric disorder. Harvard Review of Psychiatry, 17(2), 120-137. https://doi.org/10.1080/10673220902891836.

Dawson, D. N., Eisenlohr-Moul, T. A., Paulson, J. L., Peters, J. R., Rubinow, D. R., \& Girdler, S. S. (2018). Emotion-related impulsivity and rumination predict the perimenstrual severity and trajectory of symptoms in women with a menstrually related mood disorder. Journal of Clinical Psychology, 74(4), 579-593. https://doi.org/10. 1002/jclp. 22522 .

Dennerstein, L., Lehert, P., \& Heinemann, K. (2012). Epidemiology of premenstrual symptoms and disorders. Menopause International, 18(2), 48-51. https://doi.org/10. 1258/mi.2012.012013.

Eggert, L., Witthöft, M., Hiller, W., \& Kleinstäuber, M. (2016). Emotion regulation in women with premenstrual syndrome (PMS): Explicit and implicit assessments. Cognitive Therapy and Research, 40(6), 747-763. https://doi.org/10.1007/s10608016-9788-7.

Epperson, C. N., Steiner, M., Hartlage, S. A., Eriksson, E., Schmidt, P. J., Jones, I., et al. (2012). Premenstrual dysphoric disorder: Evidence for a new category for DSM-5. American Journal of Psychiatry, 169(5), 465-475. https://doi.org/10.1176/appi.ajp. 2012.11081302.

Garland, E. L., Geschwind, N., Peeters, F., \& Wichers, M. (2015). Mindfulness training promotes upward spirals of positive affect and cognition: Multilevel and autoregressive latent trajectory modeling analyses. Frontiers in Psychology, 6, 15. https:// doi.org/10.3389/fpsyg.2015.00015.

Hox, J. J., Moerbeek, M., \& Van de Schoot, R. (2017). Multilevel analysis: Techniques and applications. New York, NY: Routledge.

Hsu, S. C., Liu, C. Y., \& Hsiao, M. C. (2007). A comparison of the Tridimensional Personality Questionnaire in premenstrual dysphoric disorder and major depressive disorder. Comprehensive Psychiatry, 48(4), 366-370. https://doi.org/10.1016/j. comppsych.2007.02.006.

Huffziger, S., Ebner-Priemer, U., Zamoscik, V., Reinhard, I., Kirsch, P., \& Kuehner, C. (2013). Effects of mood and rumination on cortisol levels in daily life: An ambulatory assessment study in remitted depressed patients and healthy controls. Psychoneuroendocrinology, 38(10), 2258-2267. https://doi.org/10.1016/j.psyneuen. 2013.04.014.

Huffziger, S., Reinhard, I., \& Kuehner, C. (2009). A longitudinal study of rumination and distraction in formerly depressed inpatients and community controls. Journal of Abnormal Psychology, 118(4), 746-756. https://doi.org/10.1037/a0016946.

Jimenez, S. S., Niles, B. L., \& Park, C. L. (2010). A mindfulness model of affect regulation and depressive symptoms: Positive emotions, mood regulation expectancies, and selfacceptance as regulatory mechanisms. Personality and Individual Differences, 49(6), 645-650. https://doi.org/10.1016/j.paid.2010.05.041.

Kircanski, K., Thompson, R. J., Sorenson, J., Sherdell, L., \& Gotlib, I. H. (2018). The everyday dynamics of rumination and worry: Precipitant events and affective consequences. Cognition \& Emotion, 32(7), 1424-1436. https://doi.org/10.1080/ 02699931.2017.1278679.

Kleinstauber, M., Schmelzer, K., Ditzen, B., Andersson, G., Hiller, W., \& Weise, C. (2016). Psychosocial profile of women with premenstrual syndrome and healthy controls: A comparative study. International Journal of Behavioral Medicine, 23(6), 752-763. https://doi.org/10.1007/s12529-016-9564-9.

Kuehner, C., Welz, A., Reinhard, I., \& Alpers, G. W. (2017). Lab meets real life: A laboratory assessment of spontaneous thought and its ecological validity. PloS One, 12(9), e0184488. https://doi.org/10.1371/journal.pone.0184488.

Lanza di Scalea, T., \& Pearlstein, T. (2019). Premenstrual dysphoric disorder. Medical Clinics of North America, 103(4), 613-628. https://doi.org/10.1016/j.mcna.2019.02. 007.

Lustyk, M. K. B., Gerrish, W. G., Douglas, H., Bowen, S., \& Marlatt, G. A. (2011). Relationships among premenstrual symptom reports, menstrual attitudes, and mindfulness. Mindfulness, 2(1), 37-48. https://doi.org/10.1007/s12671-011-0041-x.

Lyubomirsky, S., Layous, K., Chancellor, J., \& Nelson, S. K. (2015). Thinking about rumination: The scholarly contributions and intellectual legacy of susan nolen-hoeksema. Annual Review of Clinical Psychology, 11, 1-22. https://doi.org/10.1146/ annurev-clinpsy-032814-112733.

Marjoribanks, J., Brown, J., O'Brien, P. M., \& Wyatt, K. (2013). Selective serotonin reuptake inhibitors for premenstrual syndrome. Cochrane Database of Systematic Reviews (6), Cd001396. https://doi.org/10.1002/14651858.CD001396.pub3.

Miller, A., Vo, H., Huo, L., Roca, C., Schmidt, P. J., \& Rubinow, D. R. (2010). Estrogen receptor alpha (ESR-1) associations with psychological traits in women with PMDD and controls. Journal of Psychiatric Research, 44(12), 788-794. https://doi.org/10. 1016/j.jpsychires.2010.01.013.

Moberly, N. J., \& Watkins, E. R. (2008). Ruminative self-focus and negative affect: An experience sampling study. Journal of Abnormal Psychology, 117(2), 314-323. https:// doi.org/10.1037/0021-843x.117.2.314. 
Nolen-Hoeksema, S., \& Watkins, E. R. (2011). A heuristic for developing transdiagnostic models of psychopathology: Explaining multifinality and divergent trajectories. Perspectives on Psychological Science : A Journal of the Association for Psychological Science, 6(6), 589-609. https://doi.org/10.1177/1745691611419672.

Nolen-Hoeksema, S., Wisco, B. E., \& Lyubomirsky, S. (2008). Rethinking Rumination. Perspectives on psychological science. A Journal of the Association for Psychological Science, 3(5), 400-424. https://doi.org/10.1111/j.1745-6924.2008.00088.x.

Owens, S. A., \& Eisenlohr-Moul, T. (2018). Suicide risk and the menstrual cycle: A review of candidate RDoC mechanisms. Current Psychiatry Reports, 20(11), 106. https://doi. org/10.1007/s11920-018-0962-3.

Petersen, N., London, E. D., Liang, L., Ghahremani, D. G., Gerards, R., Goldman, L., et al. (2016). Emotion regulation in women with premenstrual dysphoric disorder. Archives of Women's Mental Health, 19(5), 891-898. https://doi.org/10.1007/s00737-0160634-4.

Pilver, C. E., Libby, D. J., \& Hoff, R. A. (2013). Premenstrual dysphoric disorder as a correlate of suicidal ideation, plans, and attempts among a nationally representative sample. Social Psychiatry and Psychiatric Epidemiology, 48(3), 437-446. https://doi. org/10.1007/s00127-012-0548-z.

Raes, F., Hermans, D., Williams, J. M. G., Bijttebier, P., \& Eelen, P. (2008). A “Triple W"model of rumination on sadness: Why am I feeling sad, what's the meaning of my sadness, and wish I could stop thinking about my sadness (but I can't!). Cognitive Therapy and Research, 32(4), 526-541. https://doi.org/10.1007/s10608-007-9137-y.

Read, J. R., Perz, J., \& Ussher, J. M. (2014). Ways of coping with premenstrual change: Development and validation of a premenstrual coping measure. BMC Women's Health, 14, 1. https://doi.org/10.1186/1472-6874-14-1.

Reuveni, I., Dan, R., Segman, R., Evron, R., Laufer, S., Goelman, G., et al. (2016). Emotional regulation difficulties and premenstrual symptoms among Israeli students. Archives of Women's Mental Health, 19(6), 1063-1070. https://doi.org/10.1007/ s00737-016-0656-y.

Ruscio, A. M., Gentes, E. L., Jones, J. D., Hallion, L. S., Coleman, E. S., \& Swendsen, J. (2015). Rumination predicts heightened responding to stressful life events in major depressive disorder and generalized anxiety disorder. Journal of Abnormal Psychology, 124(1), 17-26. https://doi.org/10.1037/abn0000025.

Sigmon, S. T., Schartel, J. G., Hermann, B. A., Cassel, A. G., \& Thorpe, G. L. (2009). The relationship between premenstrual distress and anxiety sensitivity: The mediating role of rumination. Journal of Rational-Emotive and Cognitive-Behavior Therapy, 27(3), 188-200. https://doi.org/10.1007/s10942-009-0100-6.

Spielberger, C., Gorsuch, R. L., \& Lushene, R. (1970). Manual for the state-trait anxiety inventory. Palo Alto, CA: Consulting Psychologists Press.

Timm, C., Rachota-Ubl, B., Beddig, T., Zamoscik, V. E., Ebner-Priemer, U., Reinhard, I., et al. (2018). Mindfulness-based attention training improves cognitive and affective processes in daily life in remitted patients with recurrent depression: A randomized controlled trial. Psychotherapy and Psychosomatics, 87(3), 184-186. https://doi.org/ 10.1159/000488862.

Trull, T. J., \& Ebner-Priemer, U. (2013). Ambulatory assessment. Annual Review of Clinical Psychology, 9, 151-176. https://doi.org/10.1146/annurev-clinpsy-050212-185510.

Tschudin, S., Bertea, P. C., \& Zemp, E. (2010). Prevalence and predictors of premenstrual syndrome and premenstrual dysphoric disorder in a population-based sample. Archives of Women's Mental Health, 13(6), 485-494. https://doi.org/10.1007/s00737010-0165-3.

Watson, D., Clark, L. A., \& Tellegen, A. (1988). Development and validation of brief measures of positive and negative affect: The PANAS scales. Journal of Personality and Social Psychology, 54(6), 1063-1070.

Weise, C., Kaiser, G., Janda, C., Kues, J. N., Andersson, G., Strahler, J., et al. (2019). Internet-based cognitive-behavioural intervention for women with premenstrual dysphoric disorder: A randomized controlled trial. Psychotherapy and Psychosomatics, 88(1), 16-29. https://doi.org/10.1159/000496237.

Welz, A., Reinhard, I., Alpers, G. W., \& Kuehner, C. (2018). Happy thoughts: Mind wandering affects mood in daily life. Mindfulness, 9(1), 332-343. https://doi.org/10. 1007/s12671-017-0778-y.

Wichers, M., Geschwind, N., Jacobs, N., Kenis, G., Peeters, F., Derom, C., et al. (2009). Transition from stress sensitivity to a depressive state: Longitudinal twin study. The British Journal of Psychiatry: The Journal of Mental Science, 195(6), 498-503. https:// doi.org/10.1192/bjp.bp.108.056853.

Wittchen, H. U., Becker, E., Lieb, R., \& Krause, P. (2002). Prevalence, incidence and stability of premenstrual dysphoric disorder in the community. Psychological Medicine, 32(1), 119-132. https://doi.org/10.1017/S0033291701004925.

Wittchen, H., Wunderlich, U., Gruschwitz, S., \& Zaudig, M. (1997). Scid: Structured clinical interview for DSM-IV Axis I disorders. Göttingen: Hogrefe.. 